\title{
HOMOLOGY MODELING AND MOLECULAR DYNAMICS STUDIES OF EC1 DOMAIN OF VE-CADHERIN TO ELUCIDATE DOCKING INTERACTION WITH CADHERIN-DERIVED PEPTIDE
}

\author{
${ }^{1,2}$ Vivitri Dewi Prasasty, ${ }^{1}$ Usman Sumo Friend Tambunan and ${ }^{3}$ Teruna Jaya Siahaan \\ ${ }^{1}$ Department of Chemistry, Faculty of Mathematics and Science, \\ University of Indonesia, Depok 16424, Indonesia \\ ${ }^{2}$ Faculty of Biotechnology, Atma Jaya Catholic University of Indonesia, Jakarta 12930, Indonesia \\ ${ }^{3}$ Department of Pharmaceutical Chemistry, School of Pharmacy, \\ The University of Kansas, Lawrence 66047, United States
}

Received 2014-05-23; Revised 2014-06-07; Accepted 2014-06-25

\begin{abstract}
VE-cadherin is a protein in the cadherin family that is found at the adherens junctions of the microvessel endothelial cells of the Blood-Brain Barrier (BBB). It is recognized as the homotypic cell adhesion molecules and there is limited structural information on how VE-cadherins mediate cell-cell adhesion. It has been shown that the EC1 domain of cadherins is important for the homophilic interactions for cell-cell adhesion. Therefore, the aims of this study are to model the structure of the EC1 domain of VE-cadherin, study its molecular dynamics properties and evaluate its interactions with cadherin peptides. In this study, the sequence alignment between EC1 domain of VE-cadherin and the template protein were conducted by CLUSTALW2 platform. The SWISS-MODEL platform performed the homology modeling of the EC1 domain of VE-cadherin structure. Structural refinement was done by using KOBAMIN. Some validation analysis platforms also were conducted included PROCHECK, VERIFY3D, ERRAT and MOLPROBITY to check the allowed residues region in Ramachandran Plot (RP) and the quality of the structure. The most favored region was found $95.5 \%$ in RP value and overall model structure quality is $71.34 \%$. Molecular Dynamics (MD) was run under CABS-FLEX to determine the flexibility of the residue index. The RMSD value of MD is $1.5 \AA$ per residue index. Eventually, molecular docking by AUTODOCK VINA was conducted to investigate protein-ligand interaction. From docking, it is found that the affinity energy is -4.8 $\mathrm{kcal} / \mathrm{mol}$ which has the most favorable binding of EC1 domain with the peptide.
\end{abstract}

Keywords: VE-Cadherin, Blood Brain Barrier, Homology Modeling, Molecular Dynamics, Molecular Docking

\section{INTRODUCTION}

The difficulty of delivering drugs to the brain is due to the presence of the Blood-Brain Barrier (BBB). The $\mathrm{BBB}$ consists of microvessel endothelial cells that deliver nutrients to the brain; however, BBB prevents most drug molecules from entering the brain to make it difficult to treat drug brain diseases. The drug molecules cannot readily partition into the cell membranes to cross the BBB. The presence of metabolic enzymes and efflux pumps (i.e., P-glycoprotein (Pgp) and Multidrug Resistance Proteins (MRPs)) inhibits drug molecules from crossing the BBB via transcellular pathway. Alternatively, drug molecules can penetrate through the paracellular pathways of the BBB; however, this pathway is limited by the presence of the tight intercellular juctions (Trivedi et al., 2012; Zheng et al., 2006). Thus, the paracellular permeation of drugs can be improved by Corresponding Author: Vivitri Dewi Prasasty, Department of Chemistry, Faculty of Mathematics and Science, University of Indonesia, Depok 16424, Indonesia; and Faculty of Biotechnology, Atma Jaya Catholic University of Indonesia, Jakarta 12930, Indonesia 
increasing the porosity of the intercellular junctions. It is hoped that modulation of cadherin-cadherin interactions in the BBB can improve brain delivery of therapeutic agents across the BBB to treat brain diseases such as Alzheimer's, Parkinson's, brain tumors, multiple sclerosis (Zheng et al., 2006).

It has been shown that E-cadherin peptides can modulate the intercellular junctions and improve the brain delivery of drugs and paracellular marker molecules in in vitro and in vivo models of the $\mathrm{BBB}$. For example, HAV6 peptide (Ac-SHAVSS-NH $\mathrm{N}_{2}$ ) derived from the EC1 domain sequence of E-cadherin enhances the permeation of ${ }^{14} \mathrm{C}$-mannitol and ${ }^{3} \mathrm{H}$-daunomycin molecules through the $\mathrm{BBB}$ using the in-situ rat brain perfusion model (Kiptoo et al., 2011). Furthermore, HAV6 peptide increases the in vivo brain delivery of GdDTPA, R800 Near-IR (NIR) dye and R800cw-PEG molecules (MW $25 \mathrm{KDa}$ ) into the brain of Balb/c mice as detected by magnetic resonance imaging and NIR imaging (On et al., 2014). These results suggested that modulation of cadherin-cadherin interactions in the adherens junction can increase the porosity of the paracellular pathway of the BBB (Prasasty et al., 2014).

Vascular Endothelial Cadherin (VE-Cadherin) is present on the BBB vascular endothelium, which is responsible for cell-cell adhesion in the intercellular adherens junctions (Brasch et al., 2011). VE-cadherin is a calcium-binding protein with five repeats at the Extracellular domain (EC1-EC5) (Nanes et al., 2012). The cytoplasmic domain of VE-cadherin interacts with catenins that are connected to the actin fiber proteins. Although the structural information of human VEcadherin is not available, the homophilic interaction are found similar between VE-cadherin and E-cadherin (Nanes et al., 2012). The hypothesis is that HAV peptide derived form E-cadherin can bind to VE-cadherin to modulate the BBB porosity. To study the binding properties of HAV peptide to the EC1 domain of VEcadherin, it is necessary to build the model structure.

In this study, a homology model of the EC1 domain of human VE-cadherin was build because of the of its crystal or NMR structure was not available. The structure of chicken VE-cadherin was used as a template to build the structure of the EC1 domain of human VEcadherin. Structural refinements and molecular dynamics simulations were conducted to find structural movement and flexibility. The resulting EC1 structure was used for docking studies with the HAV peptide using Autodock Vina program. The best interaction with the lowest affinity energy will be used to describe the potential binding properties of HAV peptide with the EC1 domain of VE-cadherin. The result will also be used to improve our understanding the mechanisms of action of HAV peptide in modulating the $\mathrm{BBB}$ in vitro and in vivo. Furthermore, the results can be used to design better molecules for modulating the BBB.

\section{MATERIALS AND METHODS}

\subsection{Homology Modeling of Human EC1 Domain of VE-Cadherin}

The sequence of human EC1 domain of VE-cadherin was selected from the universal protein resource (Uniprot) (Consortium, 2014) (entry code: P33151). The template for sequence alignment was downloaded from PDB (Bernstein et al., 1977) and used the BLASTp (Altschul et al., 1990; Mount, 2007) program provided by Uniprot with default parameters. The 3D structure of chicken EC domain 1 and 2 of VE-cadherin (Uniprot ID: Q8AYDD) was downloaded from PDB (entry ID: 3PPE) (Bernstein et al., 1977; Sussman et al., 1999) as the template structure. Domain 2 of chicken cadherin as removed using Pymol (http://www.pymol.org/citing). The homology model of EC1 domain of human VEcadherin was built with SWISS-MODEL (Arnold et al., 2006). The secondary structure of EC1 domain of human VE-cadherin was predicted using the Procheck program (Laskowski et al., 1993). The target (human EC1 domain) and template (chicken EC1 domain) sequences were aligned using the ClustalW (Goujon et al., 2010; Larkin et al., 2007; McWilliam et al., 2013) method. Subsequently, the Kobamin (Rodrigues et al., 2012) tool is employed for the 3D protein structure refinement.

\subsection{Validation of Protein Structure and Its Molecular Dynamics}

The assesment of EC1 domain structure quality is done by a number of tools to check the internal consistency and reliability of the model structure. PROCHECK (Laskowski et al., 1993) analysis, which quantifies the residues in available zones of Ramachandran plot, is used to assess the stereochemical quality of the model. ERRAT (Colovos and Yeates, 1993) tool, which finds the overall quality factor of the protein, was used to check the statistics of nonbonded interactions between different atom types. Similarly to determine the compatibility of the atomic model (3D) with its own amino acid sequence (1D), the VERIFY-3D (Eisenberg et al., 1997) program was used. The MolProbity web server (Chen et al., 2010; Davis et al., 2004) was used in the quality validation of the 3D model, which provides details of atomic contact analysis of any steric problems within the molecules. 


\subsection{Molecular Docking of Protein-Ligand Complex}

The EC1 domain structure for the molecular docking experiments was built from the SWISSMODEL (Arnold et al., 2006) homology model The EC1 domain model was generated by maintaining the interface region and removing the EC2 domain and water molecules using Discovery Studio Visualizer 2.5. The 3D structures of HAV6 (Ac-SHAVSS-NH ${ }_{2}$ ) were generated using InsightII Program (Accelrys Inc.) and their conformations were optimized by energy minimization using CVFF91 force field. The final receptor and ligand coordinates were saved as pdb files.

Molecular docking experiment is performed using Autodock Vina program (Vina, The Scripps Institute) (Trott and Olson, 2010). The AutodockTools is used to add partial charges using Gasteiger method and to arrange the polar hydrogens in the protein. The ligand is set to have flexible torsion angles at all rotatable bonds, while the protein is prepared as a rigid structure. Both protein and ligand are saved as output pdbqt files. For specific docking of HAV6 peptide onto the EC1 domain, the volume grid size was adjusted to $28 \times 28 \times 28 \AA$ in the $\mathrm{x}, \mathrm{y}$ and $\mathrm{z}$ axes, respectively, with grid-points have a space within $0.375-0.625 \AA$. The conformation search was done using the Lamarckian Genetic Algorithm (LGA) and nine conformers for docking were chosen for best hits. Using affinity energy $(\mathrm{kcal} / \mathrm{mol})$ and the RootMean-Square Deviation (RMSD) $<2.0 \AA$, score and rank are calculated while running the docking program.

\section{RESULTS}

\subsection{Homology Modeling of Human EC1 Domain of VE-Cadherin}

The CLUSTALW2 program was performed to analyse the highest score of identity and similarity to the template acquired from PDB. Approximately $71 \%$ identity and $84.2 \%$ similarity showed sequence alignment of EC1 domain of VE-cadherin with the template (Fig. 1). Homology modeling was built by superimposing the desired protein with the template (Fig. 2). The crystal structure of EC1 domain of chicken VE-cadherin was recently determined at a resolution of $2.1 \AA$ and was deposited in PDB (entry ID: 3PPE) (Brasch et al., 2011). The amino acid sequence of EC1 domain is from chicken VE-cadherin is retrieved from 1 to 203 and covers the full length of EC1 domain of human VE-cadherin.

\subsection{Structure Validation and Dynamics}

In assessing $3 \mathrm{D}$ protein structure, PROCHECK was used to check the reliability of the backbone torsion angles and the built model, which quantified the residue clusters in the available zones of Ramachandran plot, as shown in Fig. 3. Ramachandran plot analysis for the modeled EC1 domain showed $95.1 \%$ residues clustered in the most favored regions, $3.3 \%$ residues were in additional allowed regions and $1.1 \%$ residue were in generously allowed regions and $0.5 \%$ residues in disallowed regions. The summary of overall assessments were performed in Table 1. The high quality of the structure was further assessed by the PROCHECK Gfactor showed -0.22 for $\mathrm{psi} / \mathrm{phi}$ only value and -0.14 for all dihedral angles value. The quality of EC1 domain model was further computed by a high ERRAT score (Fig. 4) of 71.134, which indicated acceptable protein environment (Colovos and Yeates, 1993). The VERIFY$3 \mathrm{D}$ results of EC1 domain model showed 0.36 of the amino acids (amino acids in an average 3D-1D score of $>0.2$ showed the good quality of $3 \mathrm{D}$ model) and the Molprobity score was 37.71 showed positive scores of the residues (cutoff score $>0$ ), indicating the reliability of the proposed model. The structure dynamics was performed by using CABS-FLEX platform, showing the average cluster of Root Mean Square Deviation (RMSD) value was $1.5 \AA$ (Fig. 5a). The global distance test (GDT) or GDT Total Score (GDT TS) is calculated as the largest set of amino acid residues alpha carbon atoms in the model structure falling within a defined distance cutoff of their position in the experimental structure. The GDT TS score was 1.0. The fluctuation also known as Root Mean Square Fluctuation of per residue index is depicted in Fig. 5b. The average flexibility pattern of EC1 domain showed score below 20, which is acceptable value for structural dynamic stability.

\subsection{Molecular Docking of Protein-Peptide Complex}

Molecular docking of EC1 domain-HAV peptide had been performed with Autodock Vina (Trott and Olson, 2010). From docking result, nine conformers had been selected to have high score and rank. From the nine conformers, one conformer with the lowest affinity energy value $-4.8 \mathrm{kcal} / \mathrm{mol}$, had been selected for further analysis. The binding mode of selected HAV peptide conformer with the EC1 domain is depicted in Fig. 6.

In the interaction showed that two hydrogen bonds were involved as depicted in green dash line in Fig. 7. The hydrogen bonds are contributed first from hydrogen atom from serine 1 residue of HAV peptide and oxygen from carboxylic group from residue Cysteine 23 of EC1 domain. 
Vivitri Dewi Prasasty et al. / OnLine Journal of Biological Sciences 14 (2): 155-162, 2014

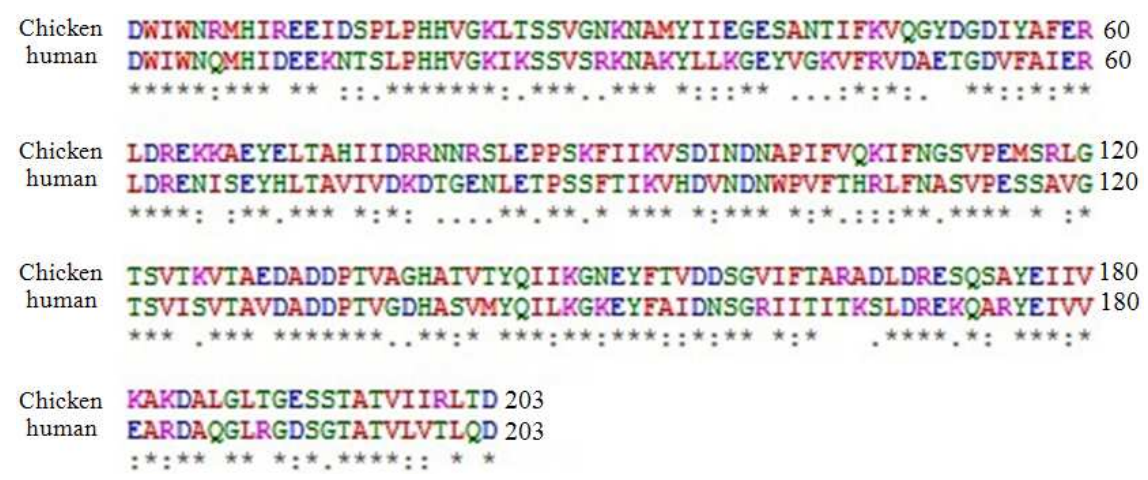

Fig. 1. Sequence alignment between chicken and human EC1 domain of VE-cadherin. The red, blue, magenta, green and grey colors indicate: hydrophobic (include aromatic), acidic, basic, hydroxyl+sulphydryl+amine and unusual amino or imino acids, respectively

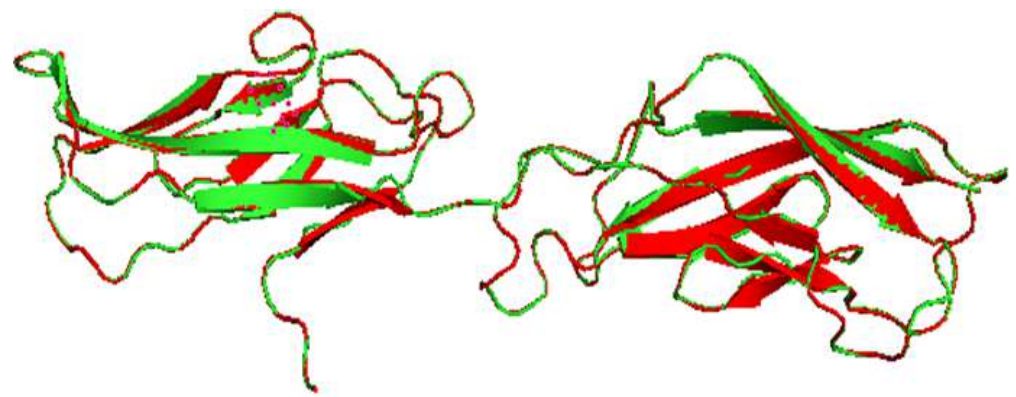

Fig. 2. The superimposed 3D EC1 domain structures. Green and red colors indicate chicken EC1 domain and human EC1 domain structure models, respectively

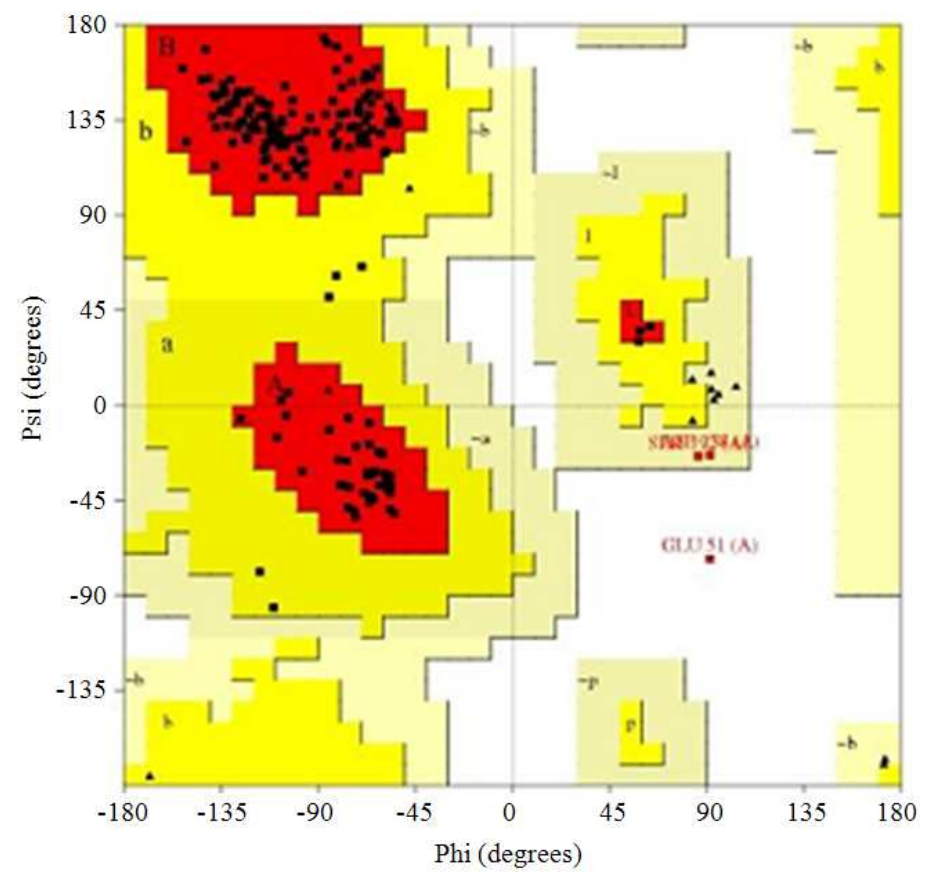

Fig. 3. Ramachandran Plot of EC1 domain of human VE-cadherin 


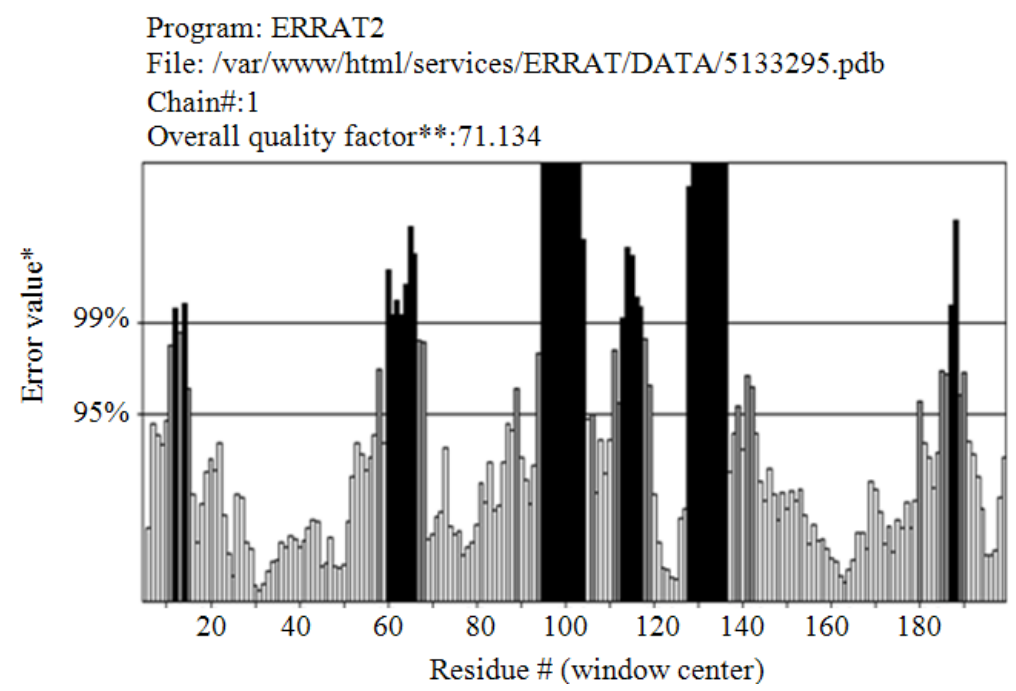

Fig. 4. The graph of error value in EC1 domain residues using ERRAT program

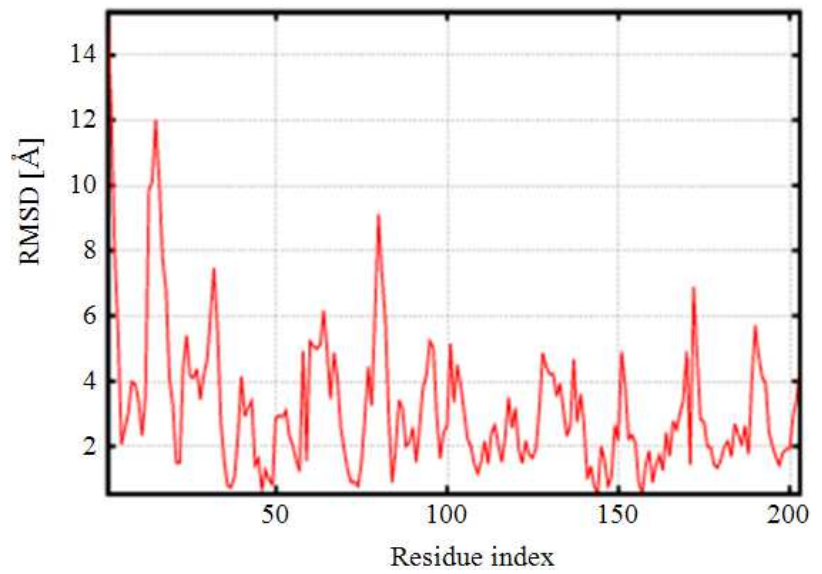

(a)

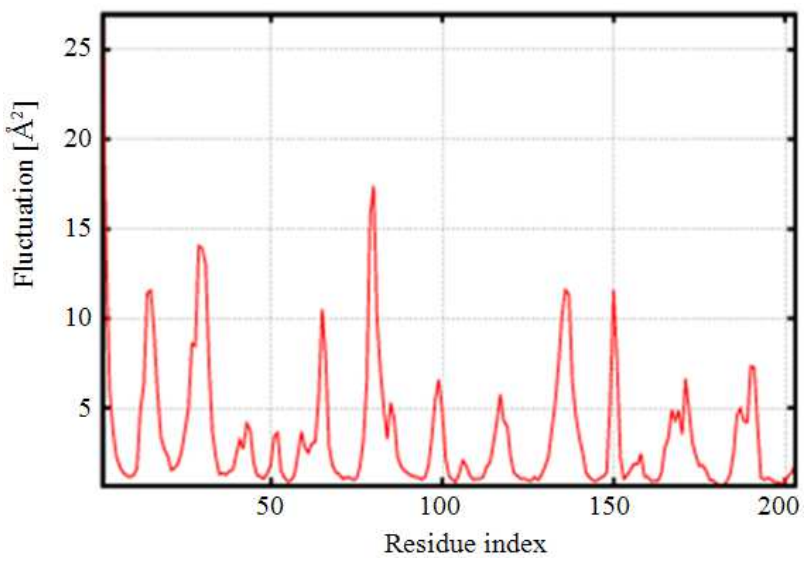

(b)

Fig. 5. (a). RMSD value of single structure mode. (b). Fluctuation value of total dynamics per residue

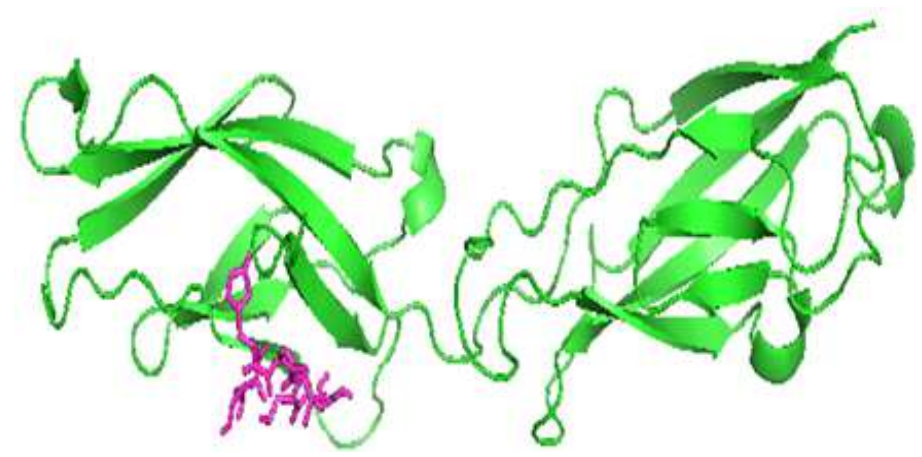

Fig. 6. Binding mode between EC1 (green) domain and HAV peptide (magenta) 


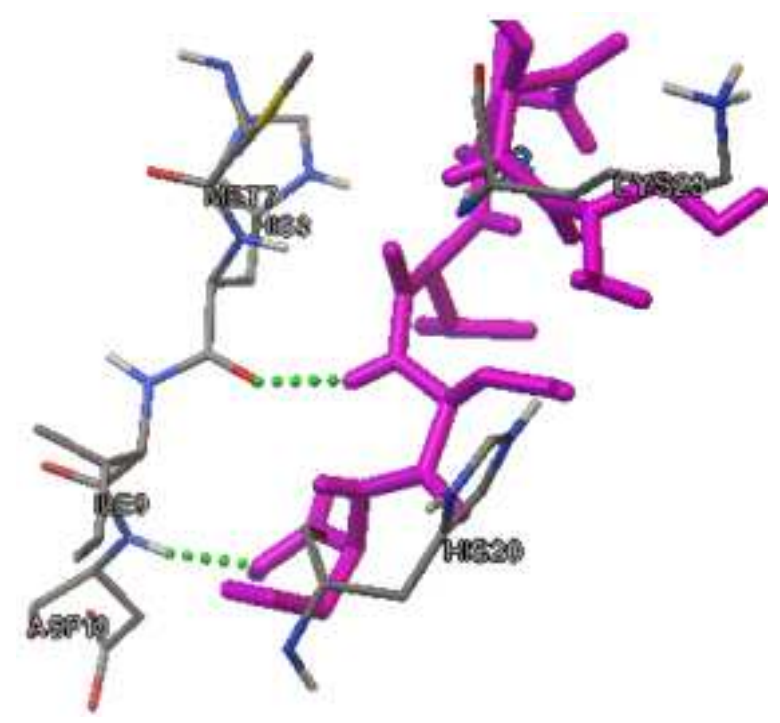

Fig. 7. Complex interaction showing hydrogen bond involved. Magenta color is HAV, multicolor is EC1 domain protein

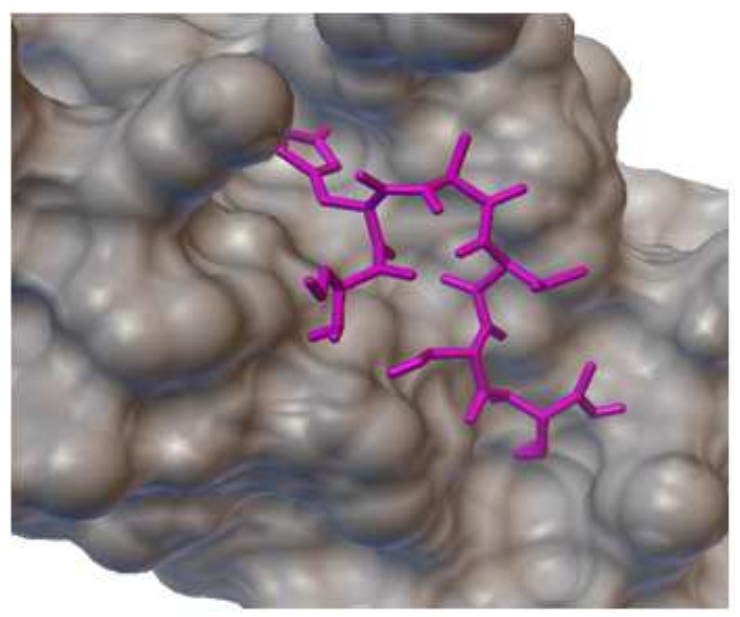

Fig. 8. The EC1 domain (grey) with the HAV peptide (magenta) docked in bulk sphere showing binding pocket

The second hydrogen bond is contributed from nitrogen atom of amide group from Aspartic acid 10 of EC1 domain with hydrogen atom of carboxylic group from residue Valine 23 of EC1 domain. The EC1 domain-HAV peptide docked in bulk sphere showing binding pocket as depicted in Fig. 8. Residue Histidine 2 of HAV peptide showed buried inside the EC1 pocket. It might induce hydrophobic interaction between the imizadole ring that is belonged to Histidine 2 of HAV peptide.
Table 1. Summary of 3D model structure assessment

\begin{tabular}{ll}
\hline Number of structures used: & 1 \\
Structure quality factors-overall statistics: & Mean score \\
Procheck G-factor e (phi/psi only) & -0.22 \\
Procheck G-factor e (all dihedral angles) & -0.14 \\
Verify3D & 0.36 \\
MolProbity clashscore & 37.71 \\
Ramachandran plot summary (Procheck) & \\
Most favoured regions (red) & $95.1 \%$ \\
Additionally allowed regions (yellow) & $3.3 \%$ \\
Generously allowed regions (light yellow) & $1.1 \%$ \\
Disallowed regions (white) & $0.5 \%$ \\
Ramachandran plot statistics (Richardson's lab) & \\
Most favoured regions & $96.5 \%$ \\
Allowed regions & $3.0 \%$ \\
Disallowed regions & $0.5 \%$ \\
\hline
\end{tabular}

\section{DISCUSSION}

Homology model of human EC1 domain of VEcadherin has extensively used to develop protein structure and function analysis. By modeled protein development, it is necessary to validate the EC1 domain model to ensure the good quality of its structure. The EC1 domain of VE-cadherin showed the high similarity with the template structure and high score in determining good quality of protein model after conducting energy minimization. Molecular dynamics simulation was used to find how much is the residue flexibility in terms of finding the structure stability. Eventually, this high quality structure further utilized for investigating the protein binding interaction with HAV peptide.

HAV peptide was first discover from the groove region of EC1 domain of E-cadherin (Sinaga et al., 2002), however the binding location might be found in multiple sites. HAV peptide containing His-Ala-Val sequence is very important to improve the paracellular porosity of the cadherin-cadherin interaction (Sinaga et al., 2002). Even though HAV peptide was derived from EC1 domain of E-cadherin, this peptide also can inhibit the cadherin interaction of Neural cadherin (Yu, 2011), other classical cadherin superfamily. Therefore, extensive study to determine HAV interaction with other type of classical peptide such as VE-cadherin is strongly suggested. From docking study, the binding mode of the complex EC1 domainHAV peptide was found in binding pocket of EC1 domain. Furthermore, this binding interaction could be a starting point and complementary information for further binding studies of VE cadherin with the HAV peptide and other potential cadherin-derived peptides using CD, NMR and Fluorescence spectroscopies. In 
the future, these conformational models will be used to design potent modulator of the tight junctions to deliver drug to the brain.

\section{CONCLUSION}

The homology modeling of human EC1 domain of VE cadherin was successfully built with high quality score. The calculated validation of the EC1 model has suggested acceptable model for further structure and function analysis such as molecular dynamics and docking interaction. From docking result, we also had successfully examined the most favorable binding mode of EC1-HAV interaction with the lowest energy affinity. This finding would give an advantage to investigate HAV peptide improvement in modulating cadherin-cadherin interaction of VE-cadherin in paracellular pathway of the BBB.

\section{ACKNOWLEDGEMENT}

This research is supported by a grant from BPPS of Indonesian Directorate General of Higher Education (DIKTI).

\section{REFERENCES}

Altschul, S.F., W. Gish, W. Miller, E.W. Myers and D.J. Lipman, 1990. Basic local alignment search tool. J. Molecular Biol., 215: 403-410. DOI: 10.1016/S0022-2836(05)80360-2

Arnold, K., L. Bordoli, J. Kopp and T. Schwede, 2006. The SWISS-MODEL workspace: A web-based environment for protein structure homology modelling. Bioinformatics 22: 195-201. DOI: 10.1093/bioinformatics/bti770

Bernstein, F.C., T.F. Koetzle, G.J. Williams, E.F. Meyer and M.D. Brice et al., 1977. The protein data bank. A computer-based archival file for macromolecular structures. Eur. J. Biochem., 80: 319-324. PMID: 923582

Brasch, J., O.J. Harrison, G. Ahlsen, S.M. Carnally and R.M. Henderson et al., 2011. Structure and binding mechanism of vascular endothelial cadherin: A divergent classical cadherin. J. Molecular Biol., 408: 57-73. DOI: 10.1016/j.jmb.2011.01.031

Chen, V.B., W.B. Arendall, J.J. Headd, D.A. Keedy and R.M. Immormino et al., 2010. MolProbity: All-atom structure validation for macromolecular crystallography. Acta Crystallogr. D Biol. Crystallogr., 66: 12-21. DOI: 10.1107/S0907444909042073
Colovos, C. and T.O. Yeates, 1993. Verification of protein structures: Patterns of nonbonded atomic interactions. Protein Sci., 2: 1511-1519. DOI: 10.1002/pro.5560020916

Consortium, T.U., 2014. Activities at the Universal Protein Resource (UniProt). Nucleic Acids Res., 42: D191-D198. DOI: 10.1093/nar/gkt1140

Davis, I.W., L.W. Murray, J.S. Richardson and D.C. Richardson, 2004. MOLPROBITY: Structure validation and all-atom contact analysis for nucleic acids and their complexes. Nucleic Acids Res., 32: W615-619. DOI: 10.1093/nar/gkh398

Eisenberg, D., R. Luthy and J.U. Bowie, 1997. VERIFY3D: Assessment of protein models with three-dimensional profiles. Meth. Enzymol., 277: 396-404. DOI: 10.1016/S0076-6879(97)77022-8

Goujon, M., H. McWilliam, W. Li, F. Valentin and S. Squizzato et al., 2010. A new bioinformatics analysis tools framework at EMBL-EBI. Nucl. Acids Res., 38: W695-W699. DOI: 10.1093/nar/gkq313

Kiptoo, P., E. Sinaga, A.M. Calcagno, H. Zhao and N. Kobayashi et al., 2011. Enhancement of drug absorption through the blood-brain barrier and inhibition of intercellular tight junction resealing by E-cadherin peptides. Mol. Pharm., 8: 239-249. DOI: 10.1021/mp100293m

Larkin, M.A., G. Blackshields, N.P. Brown, R. Chenna and P.A. McGettigan et al., 2007. ClustalW and ClustalX version 2. Bioinformatics 23: 2947-2948. DOI: 10.1093/bioinformatics/btm404

Laskowski, R.A., M.W. Macarthur, D.S. Moss and J.M. Thornton, 1993. PROCHECK: A program to check the stereochemical quality of protein structures. J. Appl. Cryst., 26: 283-291. DOI: 10.1107/S0021889892009944

McWilliam, H., W. Li, M. Uludag, S. Squizzato and Park et al., 2013. Analysis tool web services from the EMBL-EBI. Nucleic Acids Res., 41: W597-600. DOI: $10.1093 /$ nar/gkt376

Mount, D.W., 2007. Using the Basic Local Alignment Search Tool (BLAST).

Nanes, B.A., C. Chiasson-MacKenzie, A.M. Lowery, N. Ishiyama and V. Faundez et al., 2012. p120-catenin binding masks an endocytic signal conserved in classical cadherins. J. Cell Biol., 199: 365-380. DOI: $10.1083 /$ jcb.201205029

On, N.H., P. Kiptoo, T.J. Siahaan and D.W. Miller, 2014. Modulation of blood-brain barrier permeability in mice using synthetic e-cadherin peptide. Mol. Pharmaceut., 11: 974-981. DOI: $10.1021 / \mathrm{mp} 400624 \mathrm{v}$ 
Prasasty, V.D., M.E. Krause, U.S.F. Tambunan, A. Anbanandam and J.S. Laurence et al., 2014. ${ }^{1} \mathrm{H},{ }^{13} \mathrm{C}$ and ${ }^{15} \mathrm{~N}$ backbone assignment of the EC-1 domain of human E-cadherin. Biomolecular NMR Assignments. DOI: 10.1007/s12104-013-9539-6

Rodrigues, J.P., M. Levitt and G. Chopra, 2012. KoBaMIN: A knowledge-based minimization web server for protein structure refinement. Nucleic Acids Res., 40: W323-328. DOI: 10.1093/nar/gks376

Sinaga, E., S.D. Jois, M. Avery, I.T. Makagiansar and U.S. Tambunan et al., 2002. Increasing paracellular porosity by E-cadherin peptides: discovery of bulge and groove regions in the EC1-domain of Ecadherin. Pharmaceutical Res., 19: 1170-1179. DOI: 10.1023/A:1019850226631

Sussman, J.L., E.E. Abola, D. Lin, J. Jiang and N.O. Manning et al., 1999. The protein data bank. Bridging the gap between the sequence and $3 \mathrm{D}$ structure world. Genetica, 106: 149-158. DOI: 10.1023/A:1003753517358
Trivedi, M., J.S. Laurence, T.D. Williams, C.R. Middaugh and T.J. Siahaan, 2012. Improving the stability of the EC1 domain of E-cadherin by thiol alkylation of the cysteine residue. Int. J. Pharm., 431: 16-25. DOI: 10.1016/j.ijpharm.2012.03.051

Trott, O. and A.J. Olson, 2010. AutoDock Vina: Improving the speed and accuracy of docking with a new scoring function, efficient optimization and multithreading. J. Comput. Chem., 31: 455-461. DOI: $10.1002 /$ jcc. 21334

Yu, X., 2011. Tools for studying the role of N-cadherin mediated extracellular interaction in neuronal development and function. Cell Adhes. Migrat., 5: 227-231. DOI: 10.4161/cam.5.3.14776

Zheng, K., M. Trivedi and T.J. Siahaan, 2006. Structure and function of the intercellular junctions: Barrier of paracellular drug delivery. Current Pharmaceutical Design, 12: 2813-2824. DOI: $10.2174 / 138161206777947722$ 